\title{
Article \\ Asymmetric Hydrogenation of Acetophenone by Rh(I)-BINAP Supported on Smectites with Various Interlayer Distances
}

\author{
Shogo SHIMAZU, Masayuki SUZUKI, Nobuyuki ICHIKUNI, and Takayoshi UEMATSU \\ Department of Materials Engineering, Chiba University, 1-33 Yayoi, Inage, Chiba 263-8522, Japan
}

(Manuscript submitted February 15, 2003; accepted April 8, 2003)

\begin{abstract}
A new type of supported chiral rhodium catalysts (denoted as MMS) was synthesized by using smectites as hosts with various interlayer distances. The clearance spaces of smectites varied from 1.55 to $2.75 \mathrm{~nm}$ depending on the intercalated quaternary alkyl ammoniums, $\left(n-\mathrm{C}_{n} \mathrm{H}_{2 n+1}\right)_{2}\left(\mathrm{CH}_{3}\right)_{2} \mathrm{~N}^{+}(q-\mathrm{Cn} ; n=10,14$, and 18). Each MMS shows a single phase of the layer structure, suggesting that the rhodium complex and alkylammoniums exist in the same gallery. The charge density of the hosts exerts the great influence on the orientation of guests and the interlayer distance. In the hydrogenation of acetophenone, the activity increased especially with the basal spacings of smectites and the highest selectivity was 59\%e.e. achieved by Rh-BINAP/taeniolite. The open space in the gallery of our catalysts affects the activity and selectivity.
\end{abstract}

\section{Introduction}

Recently the design of new catalysts and catalytic systems has been aimed at attaining the dual goals of environmental and economic benefit.[1] A great deal of effort has been made to optimize factors such as activity, selectivity, stability, turnover number, solubility, and ease of separation from the products. Misono indicates the importance of solid catalysts from the viewpoint of atom efficiency and life cycle assessment.[2] The synthetic processes for fine chemicals and pharmaceuticals produce an extremely large number of byproducts compared with those for oil refining and bulk chemicals since the former products require the elaborate and multi-step reaction processes. In the industry of these sophisticated chemicals, high-performance catalysts with high activity and selectivity have been long sought. We have found that various supported metal complex catalysts by using clays [3-5] and MCM-41 [6] as host compounds showed the selective catalytic behavior in various organic reactions.

In this work, we have synthesized a new type of supported chiral rhodium complex catalysts showing the higher activity and asymmetric selectivity in acetophenone hydrogenation compared with the corresponding rhodium complex catalyst in homogeneous system. Our novel catalysts have large interlayer spaces by introducing quaternary alkylammoniums as co-guest molecules in order to increase the basal spacings. The relationship between the open space in the gallery of MMS and the catalytic behavior is discussed to elucidate the suitable reaction space. 


\section{Experimental}

\subsection{Materials}

Synthetic sodium hectorite (NaHT) and lithium taeniolite (LiTN) both obtained from Topy Industries Ltd. were used as host materials (cation exchange capacity (CEC): $86.6 \mathrm{meq}$ (100g-clay) $)^{-1}$ for NaHT and 268.2 meq $(100 \text { g-clay })^{-1}$ for LiTN). (R)-(+)-2,2'-Bis(diphenylphosphono)-1,1'-binaphthl ((R)-BINAP) and 1,5-cyclooctadiene (COD) were purchased from Kanto Kagaku. [Rh((R)-BINAP)(COD)]ClO 4 (Rh-BINAP) was synthesized according to the published paper.[7] Quaternary alkylammoniums with different longitudinal straight chains were used as structural tuning guests, i.e., $\left(n-\mathrm{C}_{10} \mathrm{H}_{21}\right)_{2}\left(\mathrm{CH}_{3}\right)_{2} \mathrm{NBr}, \quad\left(n-\mathrm{C}_{14} \mathrm{H}_{29}\right)_{2}\left(\mathrm{CH}_{3}\right)_{2} \mathrm{NBr}$, and $\left(n-\mathrm{C}_{18} \mathrm{H}_{37}\right)_{2}\left(\mathrm{CH}_{3}\right)_{2} \mathrm{NBr}$ were obtained from Tokyo Chemical Industry Co., Ltd. Each structural tuning guest is designated as $q-\mathrm{C}_{10}, q-\mathrm{C}_{14}$, and $q-\mathrm{C}_{18}$, respectively.

\subsection{Instruments}

IR spectra of the intercalation compounds were measured by a JEOL JIR-7000 spectrometer. XRD data were obtained by a BRUKER AXS MXP ${ }^{3} \mathrm{~V}$ powder diffractometer using Ni filtered $\mathrm{Cu} \mathrm{K} \alpha$ radiation. Loading amounts of structural tuning guests and Rh-BINAP were estimated from CHN analyses by a Perkin-Elmer-2400 and UV-Vis spectroscopy by a SHIMADZU UV-2101PC spectrometer.

\subsection{Preparation of MMS catalysts}

Multiple modified smectites (MMS) were prepared by the intercalation of Rh-BINAP into $q-\mathrm{C}_{n}(n=10,14$, and 18) modified smectites ( $q-\mathrm{C}_{\mathrm{n}}$ /host species) by cation exchange.[4(c)] The MMS catalysts are designated as $\mathrm{x} / \mathrm{y} / \mathrm{smectite}(n)$, where $x$ and $y$ indicate the loading amounts (mmol-100g) of Rh-BINAP and $q-\mathrm{C}_{\mathrm{n}}$, respectively, and $n$ is the carbon number of longitudinal alkyl chain in $q$-Cn.

\subsection{Asymmetric hydrogenation of acetophenone}

A catalyst, acetophenone, and $3 \mathrm{ml}$ of methanol were placed in an autoclave under argon atmosphere, charged with $\mathrm{H}_{2}$ at the prescribed pressure and $303 \mathrm{~K}$. The hydrogenated products were analyzed by GC-MS equipped with Rt- $\beta$-DEXsa capillary column ( $30 \mathrm{~m}, 0.25 \mathrm{~mm}$ ID).

\section{Results and Discussion}

\subsection{X-ray diffraction analysis}

The XRD parameters of the modified smectites are listed in Table 1. Each of the modified clays shows a single phase of the expanded layer structure, indicating that Rh-BINAP and structural tuning guests were present in the same gallery without segregation. The clearance space (C.S.) depended on the molecular size of $q-\mathrm{Cn}$ and the type of hosts. According to the loading
Table 1 XRD parameters ${ }^{a}$ and loading amounts of smectites and the modified smectites

\begin{tabular}{ccc}
\hline Sample $^{\mathrm{b}}$ & C.S. $/ \mathrm{nm}^{\mathrm{c}}$ & Tilting angle /degree $^{\mathrm{d}}$ \\
\hline $11.5 / 68.8 / \mathrm{NaHT}(10)$ & 1.55 & 65 \\
10.0/60.7/NaHT(14) & 2.10 & 63 \\
9.09/69.0/NaHT(18) & 2.54 & 77 \\
\hline $23.8 / 23.7 / \mathrm{LiTN}(10)$ & 2.02 & 90 \\
$23.5 / 24.6 / \mathrm{LiTN}(14)$ & 2.39 & 90 \\
$24.8 / 23.5 / \mathrm{LiTN}(18)$ & 2.75 & 90 \\
\hline
\end{tabular}

${ }^{\mathrm{a}} \mathrm{CuK \alpha} .{ }^{\mathrm{b}} \mathrm{CEC}$ is 86.6 meq (100g-clay) ${ }^{-1}$ for NaHT and $268.2 \mathrm{meq}-$ $(100 \mathrm{~g} \text {-clay })^{-1}$ for LiTN. The modified clay is denoted as $x / y /$ clay $(n)$ where $x$ and $y$ are the loading amounts (mmol (100g-clay $\left.)^{-1}\right)$ of Rh-BINAP and $q$-Cn. ${ }^{c}$ C.S. $=\mathrm{d}_{001}-0.96 \mathrm{~nm}$. The C.S. sizes of NaHT and LiTN are 0.28 and $0.22 \mathrm{~nm}$, respectively. ${ }^{\mathrm{d}}$ Elevation angle of $q-\mathrm{Cn}$ longitudinal straight chain to the layer plane. 
amounts and C.S., it can be concluded that the single layer of $q-\mathrm{Cn}$ is formed between the silicate planes. From these data, we calculated the tilting angles of $q$-Cn for MMS catalysts (Table 1). It is clear that the orientation of $q$-C $n$ depended on clay, i. e., the hectorite derivatives gave the angle ranging from 63 to $77^{\circ}$, while the taeniolites gave only $90^{\circ}$.[8] It can be explained that the clays having higher charge density tends to array guest cations with smaller occupied area parallel to silicate plane, thus enlarging the tilting angle of $q$-Cn.

\subsection{Asymmetric hydrogenation of acetophenone with various additives}

Rh-BINAP did not show the asymmetric selectivity in the hydrogenation of acetophenone (run 1 in Table 2). To enhance the chirality of Rh-BINAP, several chiral amines were examined for the catalytic reaction with or without $\mathrm{KOH}$. The results clearly showed that 1, 2-diphenylethylenediamine (DPEN) was the most effective chiral amine and the configuration of the product was mainly determined by DPEN instead of BINAP ligand (see runs 6 and 7 in Table 2). It is also found that $\mathrm{NaOH}$ remarkably accelerated the catalytic reaction. The similar results were Table 2 Asymmetric hydrogenation of acetophenone by Rh-BINAP with various additives ${ }^{\text {a }}$ published by Rossi et al. who explained the in-

\begin{tabular}{clcc}
\hline Entry & \multicolumn{1}{c}{ Additive } & $\begin{array}{c}\text { Rate/ } \\
\text { mol }(\mathrm{mol}-\mathrm{Rh} \mathrm{h})^{-1}\end{array}$ & $\%$ e.e. \\
\hline 1 & none & 0.0832 & 0.0 \\
2 & $(R, R)$-DPEN & 0.150 & 0.0 \\
3 & KOH & 1.00 & $1.4(R)$ \\
4 & $(S)-1,1^{\prime}$ '-Binaphthyl-2,2'-diamine + KOH & 1.47 & $2.4(R)$ \\
5 & L-(-)-1-Phenylethylamine + KOH & 0.431 & $5.3(R)$ \\
6 & $(R, R)$-DPEN + KOH & 1.40 & $17.3(S)$ \\
7 & $(S, S)$-DPEN + KOH & 1.46 & $27.1(R)$ \\
\hline
\end{tabular}

${ }^{a}$ Reaction conditions: reaction time, $48 \mathrm{~h} ; \mathrm{H}_{2}$ pressure, $3.03 \mathrm{MPa}$; solvent, $\mathrm{MeOH}$; bases $/ \mathrm{KOH} / \mathrm{Rh}=1.1 / 2 / 1$.

tones.[9]

\subsection{Asymmetric hydrogenation of acetophenone by MMS}

The results of acetophenone hydrogenation by various MMS catalysts were summarized in Table 3 in comparison with those of the smectite-supported Rh-BINAP without $q$-Cn (see runs 1 and 2 in Table 3). It is obvious that the taeniolite-MMS showed notably high activity and selectivity (see runs 6-9), while the hectoriteMMS had even lower activity than the smectite-supported catalysts without $q$-Cn though the hectorite-MMS had larger C.S. than the smectite-supported catalysts without $q-\mathrm{Cn}$. These results indicate that $q$-Cn in hectoriteMMS might block the part of layer space to reduce the reaction rate. Therefore, we have calculated the open space $\left(\mathrm{V}_{\mathrm{s}}\right)$ in the gallery of MMS based on the C.S., and the loading amounts of Rh-BINAP and $q$-Cn. [10] The asymmetric selectivity is plotted as ordinate and Vs as abscissa (Fig. 1). The relationship between the selectivity and $\mathrm{V}_{\mathrm{s}}$ can be recognized and Table 3 Asymmetric hydrogenation of acetophenone by clay catalysts ${ }^{a}$

\begin{tabular}{|c|c|c|c|c|}
\hline Entry & Catalysts & DPEN $^{b}$ & $\begin{array}{l}\text { Rate/ } \\
\text { mol (mol-Rh h) }\end{array}$ & \%e.e. \\
\hline 1 & $25.8 / 0 / \mathrm{NaHT}$ & $(S, S)$-DPEN & 1.08 & $35.1(R)$ \\
\hline 2 & $27.4 / 0 / \mathrm{LiTN}$ & $(S, S)$-DPEN & 1.60 & $31.1(R)$ \\
\hline 3 & $11.5 / 68.8 / \mathrm{NaHT}(10)$ & $(S, S)$-DPEN & 0.289 & $31.1(R)$ \\
\hline 4 & $10.0 / 60.7 / \mathrm{NaHT}(14)$ & $(S, S)$-DPEN & 0.297 & $34.0(R)$ \\
\hline 5 & 9.09/69.0/NaHT(18) & $(S, S)$-DPEN & 0.270 & $31.9(R)$ \\
\hline 6 & 23.8/23.7/LiTN(10) & $(S, S)$-DPEN & 1.75 & $38.7(R)$ \\
\hline 7 & 23.5/24.6/LiTN(14) & $(S, S)$-DPEN & 1.74 & $52.7(R)$ \\
\hline 8 & $24.8 / 23.5 / \mathrm{LiTN}(18)$ & $(S, S)$-DPEN & 1.72 & $59.0(R)$ \\
\hline 9 & 24.8/23.5/LiTN(18) & $(R, R)$-DPEN & 1.07 & $26.5(S)$ \\
\hline
\end{tabular}

${ }^{a}$ Reaction conditions: reaction time, $48 \mathrm{~h} ; \mathrm{H}_{2}$ pressure, $3.03 \mathrm{MPa}$; solvent, $\mathrm{MeOH}$. ${ }^{b}$ The ratio of additives DPEN/KOH/Rh=1.1/2/1. 
the selectivity is independent on the type of smectites. The MMS (24.8/23.5/LiTN(18)) with the largest open space showed the highest selectivity as well as high activity. We have speculated that large open space is necessary to form a chiral center by the coordination of DPEN to Rh-BINAP as observed for the Ru catalysts. [11]

\section{References}

1) P. T. Anastas, M. M. Kirchhoff, T. C. Williamson, Appl. Catal. A: General, 221, 3-13 (2001).

2) M. Misono, Shokubai, 43, 7-11 (2001).

3) (a) S. Shimazu, T. Ishida, and T. Uematsu, J. Mol. Catal., 55, 353-360 (1989). (b) S. Shimazu, W. Teramoto, T. Iba, M. Miura, and T. Uematsu, Catal. Today, 6, 141-146

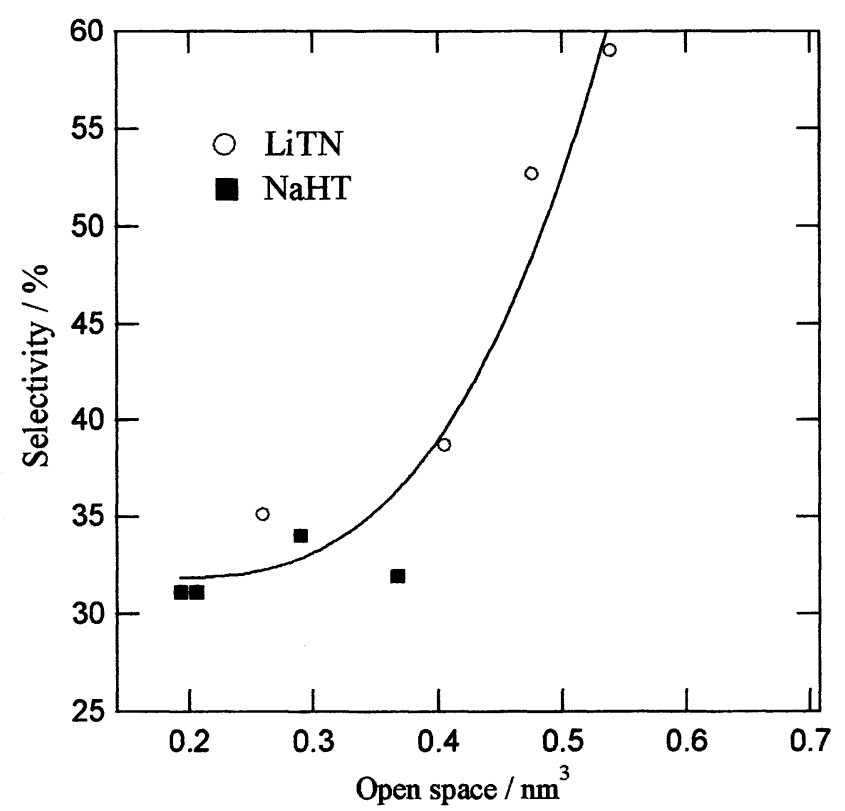

(1989). (c) S. Shimazu, W. Teramoto, and T. Uematsu, J. Mater. Sci. Lett., 8, 1368-1370 (1989). (d) S. Shimazu, W. Teramoto, and T. Uematsu, J. Ion Exchange, 4, 123-129 (1993).

4) (a) S. Shimazu, K. Ro, T. Sento, N. Ichikuni, and T. Uematsu, J. Mol. Catal. A: Chem., 107, 297-303 (1996).

(b) Sento, S. Shimazu, N. Nobuyuki, T. Uematsu, J. Mol. Catal. A: Chem., 137, 263-267 (1999). (c) T. Sento, S. Shimazu, N. Ichikuni, and T. Uematsu, Chem. Lett., 1998, 1191-1192.

5) S. Shimazu and K. Yurugi, JP Patent 285255 (2002).

6) S. Shimazu, N. Baba, N. Ichikuni, and T. Uematsu, J. Mol. Catal. A: Chem., 182-183, 343-350 (2002).

7) K. Tani, T. Yamagata, S. Akutagawa, T. Takatomi, H. Takaya, A. Miyashita, R. Noyori, and S. Otsuka, J. Am. Chem. Soc., 106, 5208-5217(1984).

8) The conformation of the alkyl chains for $q$-C $n$ in clays was detected to be all trans by ${ }^{13} \mathrm{C}$ MAS NMR studies. The results were described in the following paper: S. Shimazu M. Kawakita, N. Ichikuni, and T. Uematsu, submitted for publication.

9) M. Gargano, P. Giannoccaro, and M. Rossi, J .Organomett. Chem., 129, 239-242 (1977).

10) Open space per unit ( $a-b$ plane $\times$ clearance space) is calculated as follows: $V_{s}=V_{t}-\left(V_{q} \times x+V_{R h} \times y+V_{M^{x}}\right.$ z) where $V_{t}=$ total volume per unit $(0.52 \mathrm{~nm} \times 0.52 \mathrm{~nm} \times$ C.S. $\mathrm{nm})$ (referred from H. Shirozu, "Introduction to Clay Mineralogy", Asakura, Tokyo (1988), Chap. 2, pp.11-35), $\mathrm{V}_{\mathrm{s}}=$ Open space per unit, $\mathrm{V}_{\mathrm{q}}=$ volume of $q$-Cn (calculated from CPK model), $\mathrm{V}_{\mathrm{Rh}}=$ volume of Rh-BINAP (calculated from CPK model), $\mathrm{V}_{\mathrm{M}}=$ volume of alkali metals (calculated from ionic radius), $\mathrm{x}=$ mole ratio of $q$-Cn per unit, $\mathrm{y}=$ mole ratio of Rh-BINAP per unit, and $\mathrm{z}=$ mole ratio of alkali metal per unit.

11) R. Noyori and S. Hashiguchi, Acc. Chem. Res., 30, 97-102 (1997). 